\title{
OPEN High abundance of sugar metabolisers in saliva of children with caries
}

\author{
Muhammed Manzoor ${ }^{1}$, Sohvi Lommi ${ }^{1,2}$, Jussi Furuholm ${ }^{3}$, Catharina Sarkkola ${ }^{1}$, \\ Elina Engberg ${ }^{1,4}$, Sajan Raju ${ }^{1} \&$ Heli Viljakainen ${ }^{1,5 \bowtie}$
}

Dental caries is a biofilm-mediated, dynamic disease with early onset. A balanced salivary microbiota is a foundation of oral health, while dysbiosis causes tooth decay. We compared the saliva microbiota profiles in children with and without caries. The study consisted of 617 children aged 9-12 years from the Finnish Health in Teens (Fin-HIT) study with available register data on oral health. Caries status was summarised based on Decayed, Missing, and Filled Teeth (DMFT) index in permanent dentition. The children were then classified into the following two groups: DMFT value $\geq 1$ was considered as cavitated caries lesions (hereafter called 'caries') $(n=208)$ and DMFT $=0$ as 'cavity free' $(n=409)$. Bacterial 16S rRNA gene (V3-V4 regions) was amplified using PCR and sequenced by Illumina HiSeq. The mean age (SD) of the children was $11.7(0.4)$ years and $56 \%$ were girls. The children had relatively good dental health with mean DMFT of 0.86 (1.97). Since sex was the key determinant of microbiota composition ( $p=0.014$, we focused on sex-stratified analysis. Alpha diversity indexes did not differ between caries and cavity free groups in either sexes (Shannon: $p=0.40$ and 0.58 ; Inverse Simpson: $p=0.51$ and 0.60 , in boys and girls, respectively); neither did the composition differ between the groups ( $p=0.070$ for boys and $p=0.230$ for girls). At the genus level, Paludibacter and Labrenzia had higher abundances in the caries group compared to cavity free group in both sexes $(p<0.001)$. Taken together, there were minor differences in saliva microbiota between children with and without caries. Potential biomarkers of caries were the sugar metabolisers Paludibacter and Labrenzia. These bacteria presumably enhance salivary acidification, which contributes to progression of dental caries. The clinical relevance of our findings warrants further studies.

Human saliva consists of diverse and numerous microorganisms including bacteria, fungi, viruses, archaea, and protozoa, commonly called the saliva microbiota ${ }^{1}$. The saliva microbiota is an important part of the human microbiome. Dysbiosis in the saliva microbiota is associated with various human diseases, including obesity ${ }^{2}$, inflammatory bowel diseases ${ }^{3}$, celiac disease ${ }^{4}$, Sjögren's syndrome ${ }^{5}$, and Kostmann syndrome ${ }^{6}$.

The oral cavity is a major route into the human body, and, therefore, the saliva microbiota is vital in maintaining both systemic and oral health ${ }^{7}$. Recent evidence suggests that the normal saliva microbiota protects against colonization of pathogenic bacteria, plays a fundamental role in maintaining oral homeostasis, and prevents development of various oral diseases, including dental caries ${ }^{8-10}$. In general, the saliva microbiota is considered a good indicator of oral health ${ }^{11}$.

Dental caries is a biofilm-mediated, dynamic disease with early onset and a significant oral health problem in humans worldwide ${ }^{12,13}$. The prevalence of caries varies significantly between various parts of the world. A high prevalence is reported in many developing countries in Asia and Africa and also in some Central and Eastern European countries ${ }^{14}$. The aetiology of caries is complex and multifactorial and includes lifestyle factors (such as dietary habits, especially frequent consumption of dietary sugars), oral hygiene, use of antibiotics, fluorides, susceptible tooth surface, and biofilms ${ }^{12}$. In addition, recent evidence suggests that caries is a polymicrobial disease with some non-infectious species. However, increases in cariogenic microbial species result in tooth demineralization ${ }^{9}$. Moreover, caries is enhanced due to an ecological imbalance in the commensal microbes of the oral cavity, including sugar-fermenting and acidogenic bacteria, especially Streptococcus mutans ${ }^{15}$.

${ }^{1}$ Folkhälsan Research Center, Helsinki, Finland. ${ }^{2}$ Department of Public Health, Faculty of Medicine, University of Helsinki, Helsinki, Finland. ${ }^{3}$ Department of Oral and Maxillofacial Diseases, Faculty of Medicine, University of Helsinki, Helsinki, Finland. ${ }^{4}$ Department of Psychology and Logopedics, Faculty of Medicine, University of Helsinki, Helsinki, Finland. ${ }^{5}$ Faculty of Medicine, University of Helsinki, Helsinki, Finland. ${ }^{\square}$ email: heli.viljakainen@ helsinki.fi 


\begin{tabular}{|c|c|c|c|c|c|c|c|c|c|}
\hline & \multicolumn{3}{|c|}{ Whole sample $(\mathrm{N}=617)$} & \multicolumn{3}{|l|}{ Boys $(\mathrm{n}=272)$} & \multicolumn{3}{|l|}{ Girls $(n=345)$} \\
\hline & caries $(n=208)$ & cavity free $(n=409)$ & $p$-value & caries $(n=81)$ & cavity free $(n=191)$ & $p$-value & caries $(n=127)$ & cavity free $(n=218)$ & $p$-value \\
\hline $\begin{array}{l}\text { Age in years, median } \\
\text { (SD) }\end{array}$ & $11.7(0.3)$ & $11.8(0.4)$ & $0.051^{\varepsilon}$ & $11.7(0.4)$ & $11.7(0.4)$ & $0.302^{\varepsilon}$ & $11.7(0.4)$ & $11.7(0.3)$ & $0.079^{\varepsilon}$ \\
\hline $\begin{array}{l}\text { BMI z-score, mean } \\
(\text { SD })^{\mathrm{a}}\end{array}$ & $0.1(1.0)$ & $0.1(1.0)$ & $0.730^{\varepsilon}$ & $-0.1(1.0)$ & $0.1(1.1)$ & $0.311^{\varepsilon}$ & $0.2(0.9)$ & $0.1(0.9)$ & $0.269^{\varepsilon}$ \\
\hline \multicolumn{10}{|l|}{ Native language, $\mathbf{n}(\%)$} \\
\hline Finnish & $174(83.7)$ & $354(86.6)$ & $0.017^{\#}$ & $71(87.6)$ & $163(85.3)$ & $0.328^{\#}$ & $103(81.1)$ & $191(87.6)$ & $0.007^{\#}$ \\
\hline Swedish & $20(9.6)$ & $46(11.2)$ & & $5(6.2)$ & $21(11)$ & & $15(11.8)$ & $25(11.5)$ & \\
\hline Other & $14(6.7)$ & $9(2.2)$ & & $5(6.2)$ & $7(3.67)$ & & $9(7.1)$ & $2(0.9)$ & \\
\hline \multicolumn{10}{|c|}{ Gingival health, $\mathbf{n}(\%)$} \\
\hline No risk & $67(32.2)$ & $148(36.2)$ & $0.372^{\#}$ & $24(29.6)$ & $53(27.7)$ & $0.430^{\#}$ & $43(33.9)$ & $95(43.6)$ & $0.048^{\#}$ \\
\hline Elevated risk & $141(67.8)$ & $261(63.8)$ & & $57(70.4)$ & $138(72.3)$ & & $84(66.1)$ & $123(56.4)$ & \\
\hline \multicolumn{10}{|c|}{ Alpha diversity indexes, mean (SD) } \\
\hline Shannon & $2.92(0.29)$ & $2.92(0.28)$ & $0.613^{\epsilon}$ & $2.97(0.27)$ & $2.92(0.27)$ & $0.396^{\epsilon}$ & $2.91(0.29)$ & $2.91(0.30)$ & $0.581^{E}$ \\
\hline Inverse Simpson & $10.32(3.25)$ & $10.01(3.01)$ & $0.831^{£}$ & $10.62(2.88)$ & $10.12(3.05)$ & $0.509^{\varepsilon}$ & $10.13(3.47)$ & $9.91(2.95)$ & $0.595^{\ell}$ \\
\hline
\end{tabular}

Table 1. Comparisons performed between caries and cavity free groups for whole sample and separately for boys and girls. ${ }^{\#}$ Fisher's exact test, ${ }^{\mathfrak{E}}$ Independent samples t-test. ${ }^{\mathrm{a} D}$ Data available for $\mathrm{n}=603$. BMI $=$ body mass index, $\mathrm{SD}=$ standard deviation.

Several studies have reported that individual bacteria in saliva are positively associated with dental caries, including Streptococcus ${ }^{16}$, Lactobacilli $^{17}$, and Bifidobacteria ${ }^{18}$. Recently, the association of saliva microbiota with dental caries has been examined in adults and in the elderly in Japan, Sweden, Germany, and China ${ }^{19-22}$. However, these results were not consistent and implied that the association is complex and likely depends on the population. Some pilot studies have suggested that alterations in the saliva microbiota correlate with caries development in children ${ }^{23,24}$. However, neither age nor sex was considered in the analysis; we, and others, have demonstrated that the composition of saliva microbiota is sex-specific in children ${ }^{2,25,26}$.

To the best of our knowledge, there have been no large-scale studies that compared saliva microbiota profiles in children with and without caries. Therefore, the present study sought to investigate the composition, diversity, and abundance of saliva microbial communities in Finnish children with and without caries history in permanent dentition. The study will provide a basis for a better understanding of the microbial aetiology of caries in children and serve as the foundation for novel therapeutic strategies for caries prevention.

\section{Results}

Characteristics of the study population. The present study included 617 participants. Of these, $33.7 \%$ had a history of caries; $36.8 \%$ of girls and $29.8 \%$ of boys had experienced caries based on the register data. Participants with a history of cavitated caries lesions (hereafter called 'caries') and no history of cavitated caries (hereafter called 'cavity free') were compared with respect to sex, age, body mass index (BMI) z-score, native language, Decayed, Missing, and Filled Teeth (DMFT) index, and oral hygiene risk (Table 1). Among these, native language (Finnish, Swedish or other) differed between caries and cavity free groups $(p=0.017)$. The proportion of girls appeared higher in the caries group than in the cavity free group, however, the difference was not statistically significant $(61.1 \%$ [127/208] vs 53.3\% [218/409]; $p=0.072)$. The average (standard deviation, SD) age of participants was $11.7(0.4)$ years and ranged from 10.6 to 14.1 years. Median age was similar in the caries and cavity free group [caries $11.7(0.3)$ vs cavity free $11.8(0.4) ; p=0.051$ ]. In the entire group, the mean DMFT score was 0.86 (1.97) with minimum 0 and maximum 24. The maximum Community Periodontal Index for Treatment Needs (CPITN) value varied from 0 to 2 , and $34.8 \%$ of the children had healthy gingival tissue (hereafter called 'no oral hygiene risk') with a maximum CPITN value of 0.

Alpha and beta diversity. Alpha diversity (the diversity of microbes within a sample) indexes did not differ between caries and cavity free groups when both sexes were combined (Shannon: $p=0.613$; Inverse Simpson [Invsim]: $p=0.831$ ) (Table 1), indicating that diversity and the richness of the microbial communities were similar between groups (S1 Fig). Microbiota composition in terms of beta diversity (differences in microbial communities between two samples) was determined (Table 2$)$ firstly by sex $(p=0.014)$, secondly by gingival health $(p=0.026)$, and finally by caries $(p=0.044)$ but not by native language $(p=0.162)$. Based on these findings, we performed sex-stratified analyses in order to extract the association of caries status with saliva microbiota in homogenous subsets.

Analyses stratified by sex. Alpha and beta diversity. We compared alpha diversity indexes by caries status separately in boys and girls. In boys, Shannon and Invsim indexes did not differ between the groups ( $p=0.396$ and $p=0.509$, respectively) (Table 1). Similarly, in girls, no significant differences in alpha diversity (Shannon: $p=0.581$; Invsim: $p=0.595$ ) were observed between caries and cavity free groups (Table 1 ). There were no differences in beta diversity between caries and cavity free groups among boys $(p=0.070)$ or among girls $(p=0.230)$ (Fig. 1; Table 2). 


\begin{tabular}{|l|l|l|l|}
\hline & $\mathbf{R}^{2}$ & F-value & $\boldsymbol{p}$-value \\
\hline Whole group & & & \\
\hline Sex & 0.004 & 2.551 & 0.014 \\
\hline Gingival health & 0.003 & 2.106 & 0.026 \\
\hline Caries status & 0.003 & 2.047 & 0.044 \\
\hline Native language & 0.004 & 1.315 & 0.164 \\
\hline Sex-stratified \\
\hline Boys \\
\hline Caries status & 0.007 & 1.787 & 0.070 \\
\hline Gingival health & 0.006 & 1.489 & 0.142 \\
\hline Girls & \multicolumn{5}{|l}{} \\
\hline Caries status & 0.004 & 1.240 & 0.230 \\
\hline Gingival health & 0.005 & 1.658 & 0.105 \\
\hline
\end{tabular}

Table 2. Determinants of beta diversity in whole group, and separately in boys and girls. PERMANOVA analysis for the beta diversity using Bray Curtis dissimilarity index among the study participants adjusted for age, gender, BMI z-score, native language, and gingival health.

Differential abundance. In boys, the five top genera Rothia, Neisseria, Haemophilus, Paludibacter, and Labrenzia were more abundant in the caries group (adjusted $p$-value $<0.001$ ), while Anaerococcus $(p=0.005)$, Caulobacter $(p=0.004)$, Macrococcus $(p=0.004)$, Phenylobacterium $(p=0.004)$, and Acinetobacter $(p=0.004)$ were less abundant with caries than cavity free group (Table 3; Fig. 2a).

Similarly, in girls, the five top genera Prevotella, Selenomonas, Actinomyces, Paludibacter and Labrenzia were highly abundant in the caries group (adjusted $p$-value $<0.001$ ), while Hyphomicrobium, Bdellovibrio, Weissella, Alistipes, and Xylanibacter were less abundant with caries than cavity free group (adjusted $p$-value $<0.001$ ) (Table 4; Fig. 2b).

Taken together, the shared saliva microbes found with greater abundancy in both sexes were Paludibacter and Labrenzia in the caries group than in the cavity free group, while, Phenylobacterium, Macrococcus and Pelomonas were less abundant. On the other hand, Selenomonas showed an inconsistent abundancy in boys and girls.

\section{Discussion}

This study employed 16S rRNA amplicon sequencing to characterize the saliva microbial communities in preadolescent children with and without history of caries in permanent dentition. In total, the history of cavitated caries lesions was recorded in $34 \%$ of our participants. Girls were somewhat overrepresented in the caries group. Sex was a stronger determinant of the saliva microbiota than caries. Caries was associated with differences in the abundance of several taxa but not with overall microbial composition or diversity. Paludibacter and Labrenzia were the key caries-related taxa inhabitants both in preadolescent boys and girls, suggesting a possible pathogenic role of these genera in caries development.

Paludibacter is an anaerobic, chemoorganotrophic bacterium in the phylum Bacteroidetes. Recently, this genus was found in the subgingival plaque of periodontally healthy individuals ${ }^{29}$. Similar to other common saliva microbiota (such as Fusobacterium and Gemella), Paludibacter also helps in energy metabolism pathways and cell motility ${ }^{30}$. Labrenzia is a genus of bacteria from the phylum Proteobacteria and only a few species have been identified in this genus. Labrenzia is mainly found in soil and marine environments ${ }^{31}$. We believe the genus Labrenzia has not been linked with caries before. Along with other acid producers, Labrenzia are assumed to enhance salivary acidification, which contributes to the progression of dental caries. Paludibacter and Labren$z i a$ are the two shared saliva microbes positively associated with caries in our study both in boys and girls.

The aetiology of caries is complex and alterations in the saliva microbiota are associated with the development of caries in children ${ }^{9,23,24}$. We observed minor differences in the abundance of several taxa between caries and cavity free groups in sex-stratified analysis. Sex was the major determinant of saliva microbiota composition; we are unaware if previous studies have considered sex in their analysis. Similar to our findings, Jiang et al. ${ }^{32}$ also found minor differences in the abundance of several species in caries and cavity free groups in children (3-4 years old) without formal statistical significance. However, they did not observe any difference in salivary microbiota diversity or composition between boys and girls ${ }^{32}$. In our study, beta diversity was similar between caries and cavity free groups. In subjects 60 years and older, Jiang et al. ${ }^{22}$ observed differences in beta diversity between caries and cavity free groups which is inconsistent with our study ${ }^{22}$. However, most of previous studies included very small sample sizes and were performed at different geographic locations $s^{9,22,24,32}$. Evidently, we have conducted the largest study on saliva microbiota and the history of cavitated caries lesions in children.

The sex-stratified analysis revealed potential pathogens, suggesting similar pathogenesis of caries in girls and boys. The timing of dental development differs slightly between boys and girls ${ }^{33}$. However, our results are consistent with other studies that demonstrate that caries is more common in girls than in boys ${ }^{34,35}$. Ortiz et al. ${ }^{25}$ examined the sex-specific differences in the saliva microbiota in children with active caries and identified several species (including Rothia aeria, Neisseria flavescens, and Haemophilus pittmaniae) that were higher in boys with active caries. Our findings are in line with these observations. While the genus Rothia is a key member of the saliva microbiota in persons with caries, its role is largely unknown ${ }^{36,37}$. The majority of the Neisseria species 

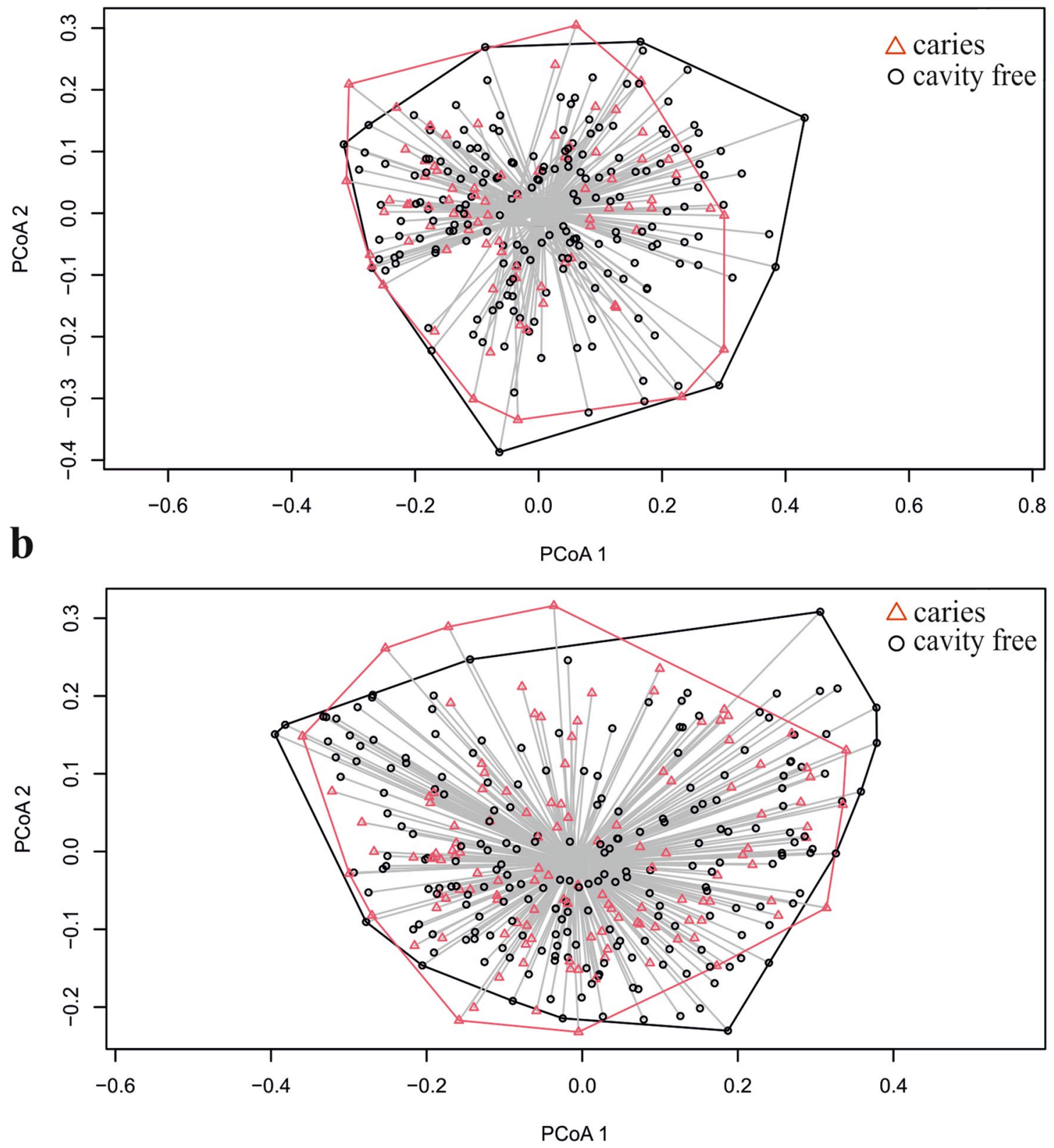

Figure 1. Principal coordinates analysis (PCoA) plot of abundance based on Bray-Curtis dissimilarity (beta diversity) of the saliva microbiota composition in caries and cavity free groups in boys (a) and girls (b).

are assumed to be acid producers and therefore enhance the development of a cariogenic environment ${ }^{25}$. We found that Haemophilus was higher in boys with caries; however, the genus Haemophilus was also reported in the saliva microbiota in those with low levels of caries ${ }^{38}$. We found that the genera Phenylobacterium, Macrococcus and Pelomonas were less abundant in the caries group than in the cavity free group in both sexes. Phenylobacterium are strictly aerobic non-motile bacteria that belong to Caulobacteraceae family, and the role of Phenylobacterium species in saliva microbiota has not been elucidated yet ${ }^{39}$. Macrococcus genus belongs to the family Staphylococcaceae, and is closely related to the genus Staphylococcus, and this genus is considered nonpathogenic ${ }^{40}$. We have previously reported that Macrococcus were less abundant in the saliva of overweight children compared with normal weight children ${ }^{2}$. Pelomonas is a genus from the family Comamonadaceae and the abundance of Pelomonas has shown to decrease with age in the microbiota of gingival crevicular fluid and tongue back in healthy individuals ${ }^{41}$. Our finding on Selenomonas is discordant between sexes. Ortiz et al. ${ }^{25}$ showed that Selenomonas sps were associated with caries in girls, and a notably greater abundance of Selenomonas Genus probe 1 was observed with less frequent teeth brushing in adolescents with caries ${ }^{20}$, which is in line with our finding in girls. Taken together, we found that most of the caries-associated taxa were acid producers that cause demineralisation of tooth enamel. Accordingly, children with a greater abundance of acid producers may be more prone to caries development. 


\begin{tabular}{|l|l|l|r|c|}
\hline OTUs & Genus & log2FoldChange & $\boldsymbol{p}$-value & Adjusted $\boldsymbol{p}$-value \\
\hline Otu000007 & Rothia & +11.094 & 0.001 & $<0.001$ \\
\hline Otu000006 & Neisseria & +10.735 & 0.001 & $<0.001$ \\
\hline Otu000008 & Haemophilus & +10.563 & $<0.001$ & $<0.001$ \\
\hline Otu000130 & Paludibacter & +1.556 & $<0.001$ & $<0.001$ \\
\hline Otu000176 & Labrenzia & +1.189 & $<0.001$ & $<0.001$ \\
\hline Otu000222 & Shuttleworthia & -1.379 & 0.010 & 0.012 \\
\hline Otu000492 & Mogibacterium & -3.329 & $<0.001$ & 0.003 \\
\hline Otu000349 & Brachymonas & -3.964 & 0.012 & 0.013 \\
\hline Otu000748 & Selenomonas & -4.228 & 0.005 & 0.006 \\
\hline Otu004001 & Stenotrophomonas & -5.577 & 0.002 & 0.004 \\
\hline Otu000804 & Pelomonas & -6.145 & 0.002 & 0.004 \\
\hline Otu001818 & Acinetobacter & -6.173 & 0.002 & 0.004 \\
\hline Otu002378 & Phenylobacterium & -6.247 & 0.002 & 0.004 \\
\hline Otu001497 & Macrococcus & -6.353 & 0.002 & 0.004 \\
\hline Otu005048 & Caulobacter & -6.577 & 0.002 & 0.004 \\
\hline Otu005348 & Anaerococcus & -7.577 & 0.004 & 0.005 \\
\hline
\end{tabular}

Table 3. List of differentially abundant genera in boys in caries group compared with cavity free group. $\log 2$ FoldChange value $(+)$ means higher and $(-)$ means lower abundancy in caries group compared with cavity free group.

In addition to oral hygiene, frequent consumption of dietary sugars is the leading cause of caries; bacteria in the oral cavity break down dietary sugars and produce acids that destroy tooth enamel, slowly leading to tooth decay $^{42}$. Our results highlight dissimilarly of saliva microbiota between sexes, while support similar taxa related to cavitated caries lesions. Tooth-brushing and eating habits were not considered here, although they may differ between girls and boys, as reported before ${ }^{20,43}$. Moreover, variation in the quality of tooth enamel, saliva flow and composition may also contribute to gender differences in saliva microbiota as well as in caries ${ }^{44,45}$. Thus, further studies are needed to address their effects on caries.

The strengths of the present study include a large sample size, the inclusion of a homogeneous age group of both sexes, and availability of primary healthcare visit data collected in dental appointments. Caries was diagnosed by a dental professional and was not self-reported as in many other studies ${ }^{46,47}$. However, our study has several limitations. First, the nature of the study is descriptive due to a cross-sectional study design. From the randomly selected 1000 saliva samples, we limited our material to 617 based on the available records on dental examinations within 12 months. The mean time difference between the saliva sampling and dental appointment was 4.4 months, which we consider a fairly representative timeframe. Our study compared groups with or without history of cavitated caries lesions, not caries activity, in relatively low caries-risk paediatric population, thus a wider timeframe was considered justified. Secondly, participants in the present study were children with late mixed or fully permanent dentition. The eruption schedule of permanent teeth is thoroughly studied and well$\mathrm{known}^{48}$; deciduous teeth are typically exfoliated at 6 to 12 years of age and during this time children have a mixture of permanent and deciduous teeth. Since records on deciduous teeth were limited, the analyses were based on permanent dentition. Hence, early caries experience in deciduous teeth was not included, which may affect our results. Moreover, information on tooth brushing, smoking and other dental hygiene habits is not known.

Dental health measured with the DMFT index has improved among Finnish children since the $1990 \mathrm{~s}^{49}$. The mean DMFT index values were 0.7 in 12 -year-olds in 2009 and our observation is in agreement with this ${ }^{49}$. For our study, the DMFT index was available as a composite variable, which is an indicator of individual's history of cavitated caries lesions rather than current caries activity. This discrepancy and the overall usability of the DMFT index has been widely discussed ${ }^{50}$, and more elaborate indexes have been suggested, such as the T-Health inde ${ }^{51}$. For periodontal or gingival health status, information was obtainable solely as CPITN index scores. Since actual diseases of the periodontium are rare in this age group, we dichotomized the variable and considered it as a reflection of the level of dental hygiene. Finally, our microbiota analysis was limited to the genus level. However, species-level identification would provide a more precise identity in terms of potential biomarkers related to caries. We demonstrated that Paludibacter and Labrenzia are the 'keystone pathogens' that influence the caries process by altering the 'healthy' microbiota to a disease state. Such observation have also been reported in periodontal disease $\mathrm{e}^{52}$.

To conclude, cavitated caries lesions in permanent dentition is associated with relatively minor changes in the saliva microbiota in preadolescents with relatively good dental health. We identified Paludibacter and Labrenzia as potential biomarkers of caries; both are sugar metabolisers. Paludibacter and Labrenzia likely enhance salivary acidification, which contributes to the progression of dental caries. However, the clinical relevance of our findings warrants further studies. 

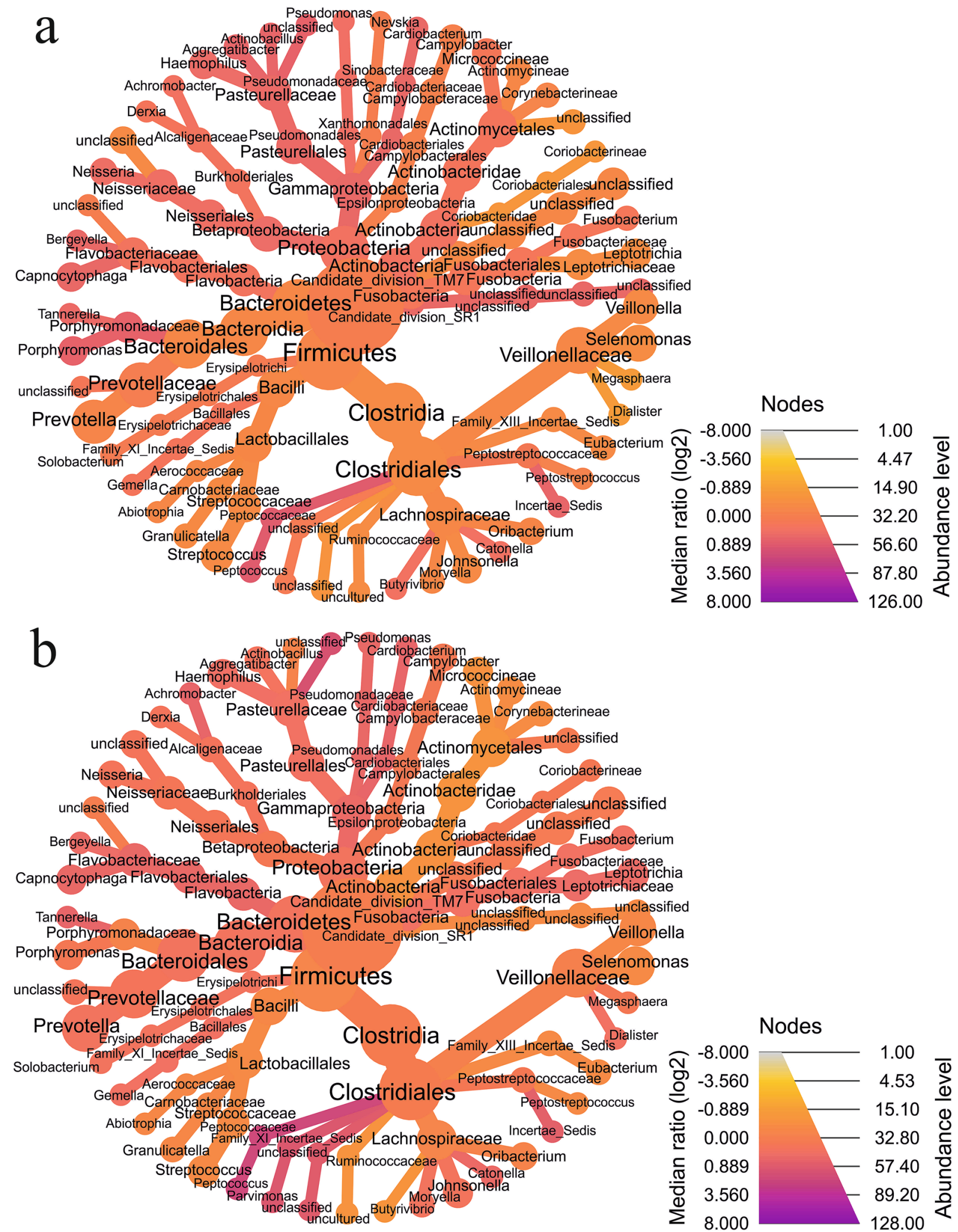

Figure 2. Heat tree analysis leverages the hierarchical structure of taxonomic classifications to quantitatively (using median abundance) and statistically (using non-parametric Wilcoxon Rank Sum test) depict taxonomic differences between microbial communities in boys (a) and girls (b). The image is drawn using web-based platform MicrobiomeAnalyst ${ }^{27}$ (https://www.microbiomeanalyst.ca/) and trees are automatically created and arranged when there are multiple roots to the taxonomy ${ }^{28}$. 


\begin{tabular}{|l|l|l|r|c|}
\hline OTUs & Genus & log2FoldChange & $\boldsymbol{p}$-value & Adjusted $\boldsymbol{p}$-value \\
\hline Otu000003 & Prevotella & +11.482 & $<0.001$ & $<0.001$ \\
\hline Otu000009 & Selenomonas & +10.168 & $<0.001$ & $<0.001$ \\
\hline Otu000033 & Actinomyces & +7.182 & $<0.001$ & $<0.001$ \\
\hline Otu000130 & Paludibacter & +1.719 & $<0.001$ & $<0.001$ \\
\hline Otu000176 & Labrenzia & +0.853 & $<0.001$ & $<0.001$ \\
\hline Otu001039 & Rhodanobacter & -3.719 & $<0.001$ & $<0.001$ \\
\hline Otu001497 & Macrococcus & -4.219 & 0.006 & 0.007 \\
\hline Otu000648 & Dolosigranulum & -4.249 & 0.001 & 0.002 \\
\hline Otu000804 & Pelomonas & -4.912 & 0.002 & 0.003 \\
\hline Otu002174 & Leuconostoc & -6.804 & $<0.001$ & 0.001 \\
\hline Otu002378 & Phenylobacterium & -6.804 & $<0.001$ & 0.001 \\
\hline Otu003167 & Xylanibacter & -6.804 & $<0.001$ & 0.001 \\
\hline Otu004423 & Alistipes & -6.804 & $<0.001$ & 0.001 \\
\hline Otu005632 & Weissella & -7.554 & $<0.001$ & 0.001 \\
\hline Otu005084 & Bdellovibrio & -7.564 & $<0.001$ & 0.001 \\
\hline Otu004975 & Hyphomicrobium & -7.627 & $<0.001$ & 0.001 \\
\hline
\end{tabular}

Table 4. List of differentially abundant genera in girls in caries group compared with cavity free group. log2FoldChange value (+) means higher and (-) means lower abundancy in caries group compared with cavity free group.

\section{Materials and methods}

Study design. This study utilized saliva samples from the Finnish Health in Teens (Fin-HIT) study, which includes approximately 11,400 children aged mainly 9-12 years. Data were collected between 2011 and 2014 mostly in schools across Finland. The study protocol has been described in detail elsewhere ${ }^{53}$. Altogether 1000 samples were randomly selected from this cohort to obtain an unbiased representation of the population. Age and native language (Finnish, Swedish or other) were reported by the participants or their parents. BMI z-score was calculated based on measured weight and height. Participants with antibiotic use during the 3 months prior to sampling ${ }^{54}(\mathrm{n}=31)$ or without sufficient information on oral health $(\mathrm{n}=255)$ were excluded. After saliva processing, 25 participants withdrew their consent. Furthermore, after processing the microbiota we found that 72 samples had low sequence depth, which may influence the diversity $(<10,000$ sequences). These samples were also excluded. The Fin-HIT study protocol was approved by the Coordinating Ethics Committee of the Hospital District of Helsinki and Uusimaa in Finland (169/13/03/00/10). Written informed consent was obtained from the children and one of their parents. All study procedures were performed in accordance with the Helsinki Declaration.

Oral health. Data on oral health variables were collected from Avohilmo maintained by the Finnish Institute for Health and Welfare (THL) (https://thl.fi/en/web/thlfi-en). Variables regarding the prevalence and history of dental caries were scores of decayed (D) permanent teeth, missing (M) permanent teeth due to caries, and filled (F) permanent teeth. The information on caries status was collected from dental appointments closest to the saliva sampling within 12 months. The mean time difference between the saliva sample collection and dentist appointment was 4.4 (SD 2.25) months. The DMFT index was summarised based on caries status scores in permanent dentition ${ }^{50,55}$, and the composite DMFT index was used as the main outcome. Children were classified into the following two groups: DMFT value $\geq 1$ was considered as 'caries' and DMFT $=0$ as 'cavity free'. The final study population consisted of 617 participants $(n=208$ for caries and $n=409$ for cavity free).

Gingival health status was registered in Avohilmo records with the CPITN as reported by Ainamo et al. ${ }^{56}$; this was used as an indicator of dental hygiene status. We categorized the study participants in two groups by their highest CPITN value; $\mathrm{CPITN}$ value $=0$ was considered as 'no oral hygiene risk' and CPITN value $1-2$ indicated 'elevated risk regarding oral hygiene'.

16S rRNA gene sequencing and processing of sequencing data. We have previously reported associations of saliva microbiota composition and diversity with weight status ${ }^{2}$, meal regularity ${ }^{57}$, breast feeding ${ }^{58}$, and lifelong antimicrobial purchases ${ }^{54}$. Saliva samples (unstimulated, up to $2 \mathrm{ml}$ ) were collected from each participant in Oragene-DNA (OG-500) self-collection kits (DNA Genotek Inc., Canada) containing a stabilizing reagent and transported to the laboratory for DNA extraction. The samples were lysed using $50 \mathrm{ml}$ lysozyme $(10 \mathrm{mg} / \mathrm{ml}$, Sigma-Aldrich), $6 \mathrm{ml}$ mutanolysin $(25 \mathrm{KU} / \mathrm{ml}$, Sigma-Aldrich), and $3 \mathrm{ml}$ lysostaphin $(4000 \mathrm{U} / \mathrm{ml}$, Sigma-Aldrich), after which a 500-ml aliquot of cell suspension was added and further incubated for $1 \mathrm{~h}$ at $37^{\circ} \mathrm{C}$. After lysis, genomic DNA was extracted using a CMG-1035 saliva kit and Chemagic MSM1 nucleic acid extraction robot (PerkinElmer ${ }^{2,54}$. 16S rRNA amplification was performed with an in-house protocol as described previously $^{59}$. The $\mathrm{V} 3-\mathrm{V} 4$ variable regions were amplified using primers reported previously ${ }^{60}$. DNA quantity 
was assessed using an Agilent 2100 Bioanalyzer and PCR products were processed for paired-end sequencing $(2 \times 270 \mathrm{bp})$ on a HiSeq1500 platform (Illumina, CA, USA).

The paired-end reads were merged together to reconstruct full-length sequences using mothur pipeline (Version v.1.35.1). Sequencing quality was performed and further processed using the MiSeq $\mathrm{SOP}^{61}$. The sequence reads containing ambiguous bases $(\mathrm{N})$, homopolymer stretches $(>8$ bases), and small reads $(<330$ bases) were removed using mothur pipeline ${ }^{62}$. UCHIME algorithm incorporated in the mothur was used to remove chimeric sequences $^{63}$. The high-quality sequence reads were then aligned to the Silva $16 \mathrm{~S}$ rRNA reference database ${ }^{64}($ Ver V119) and clustered at $>98 \%$ homology to identify Operational Taxonomic Units (OTUs). The most abundant bacterial taxa were recognized at the genus level. Alpha diversity indexes (Shannon index and Invsim index) were calculated to illustrate the microbiota diversity and richness in each sample. Beta diversity was calculated using Bray Curtis dissimilarity index. These were performed using the R-package 'vegan' (version 2.5-6) ${ }^{65}$.

Statistical analysis. The normality of the distributions was visually examined, and appropriate tests were used for the analysis. Independent samples t-test (for continuous variables) and Fisher's exact test (for categorical variables) were performed to examine differences between the caries and cavity free groups. Permutational analysis of variance (PERMANOVA) test was used to test the differences in microbial community composition between individuals with caries vs cavity free using the adonis and betadisper function in Phyloseq R-package (version 1.32.0). Differentially abundant OTUs were identified at the genus taxonomic level using DESeq2 incorporated in Phyloseq R-package (version 1.32.0). The results are presented with mean (SD) unless indicated otherwise. All statistical analyses were conducted using IBM SPSS for Windows, version 20 (IBM, Chicago, IL, USA). The statistical significance level was set at $5 \%$. $p$-values were calculated and adjusted by the false discovery rate (FDR).

\section{Data availability}

All relevant data are within the manuscript and its Supporting Information files.

Received: 29 October 2020; Accepted: 5 February 2021

Published online: 24 February 2021

\section{References}

1. Nasidze, I., Li, J., Quinque, D., Tang, K. \& Stoneking, M. Global diversity in the human salivary microbiome. Genome Res. 19, 636-643 (2009).

2. Raju, S. C. et al. Gender-Specific Associations Between Saliva Microbiota and Body Size. Front. Microbiol. 10, 767. https://doi. org/10.3389/fmicb.2019.00767 (2019).

3. Xun, Z., Zhang, Q., Xu, T., Chen, N. \& Chen, F. Dysbiosis and ecotypes of the salivary microbiome associated with inflammatory bowel diseases and the assistance in diagnosis of diseases using oral bacterial profiles. Front. Microbiol. 9, 1136. https://doi. org/10.3389/fmicb.2018.01136 (2018).

4. Francavilla, R. et al. Salivary microbiota and metabolome associated with celiac disease. Appl. Environ. Microbiol. 80, 3416-3425 (2014).

5. Rusthen, S. et al. Dysbiotic salivary microbiota in dry mouth and primary Sjögren's syndrome patients. PLoS ONE 14, e0218319 (2019).

6. Topcuoglu, N., Erdem, A. P., Karacan, I. \& Kulekci, G. Oral microbial dysbiosis in patients with Kostmann syndrome. J. Med. Microbiol. 68, 609-615 (2019).

7. Llena-Puy, C. The rôle of saliva in maintaining oral health and as an aid to diagnosis. Med. Oral. Patol. Oral. Cir. Bucal. 11, 449-455 (2006).

8. Hemadi, A. S., Huang, R., Zhou, Y. \& Zou, J. Salivary proteins and microbiota as biomarkers for early childhood caries risk assessment. Int. J. Oral Sci. https://doi.org/10.1038/ijos.2017.35 (2017).

9. $\mathrm{Xu}, \mathrm{L}$. et al. Dynamic alterations in salivary microbiota related to dental caries and age in preschool children with deciduous dentition: a 2-year follow-up study. Front. Physiol. https://doi.org/10.3389/fphys.2018.00342 (2018).

10. Pedersen, A. M. L. \& Belstrøm, D. The role of natural salivary defences in maintaining a healthy oral microbiota. J. Dent. 80, S3-12 (2019).

11. Sultan, A. S., Kong, E. F., Rizk, A. M. \& Jabra-Rizk, M. A. The oral microbiome: a Lesson in coexistence. PLOS Pathog. 14, e1006719 (2018).

12. Selwitz, R. H., Ismail, A. I. \& Pitts, N. B. Dental caries. Lancet 369, 51-59 (2007).

13. Machiulskiene, V. et al. Terminology of dental caries and dental caries management: consensus report of a workshop organized by ORCA and cariology research group of IADR. Caries Res. 54, 7-14 (2020).

14. Petersen, P. E. Challenges to improvement of oral health in the 21st century-the approach of the WHO Global Oral Health Programme. Int Dent J. 54, 329-343 (2004).

15. Metwalli, K. H., Khan, S. A., Krom, B. P. \& Jabra-Rizk, M. A. Streptococcus mutans, Candida albicans, and the human mouth: a sticky situation. PLoS Pathog. 9, e1003616 (2013).

16. Forssten, S. D., Björklund, M. \& Ouwehand, A. C. Streptococcus mutans, caries and simulation models. Nutrients 2, 290-298 (2010).

17. Nase, L. et al. Effect of long-term consumption of a probiotic bacterium, Lactobacillus rhamnosus GG, in milk on dental caries and caries risk in children. Caries Res. 35, 412-420 (2001).

18. Beighton, D. et al. Oral bifidobacteria: caries-associated bacteria in older adults. J. Dent Res. 89, 970-974 (2010).

19. Takeshita, T. et al. Bacterial diversity in saliva and oral health-related conditions: the Hisayama Study. Sci. Rep. 6, 22164. https:// doi.org/10.1038/srep22164 (2016).

20. Eriksson, L., Lif Holgerson, P. \& Johansson, I. Saliva and tooth biofilm bacterial microbiota in adolescents in a low caries community. Sci. Rep. 7, 5861. https://doi.org/10.1038/s41598-017-06221-z (2017).

21. Rupf, S. et al. Comparison of initial oral microbiomes of young adults with and without cavitated dentin caries lesions using an in situ biofilm model. Sci. Rep. 8, 14010. https://doi.org/10.1038/s41598-018-32361-x (2018).

22. Jiang, Q., Liu, J., Chen, L., Gan, N. \& Yang, D. The oral microbiome in the elderly with dental caries and health. Front. Cell Infect. Microbiol. https://doi.org/10.3389/fcimb.2018.00442 (2019).

23. Vieira, A. R. et al. Profiling microorganisms in whole saliva of children with and without dental caries. Clin Exp Dent Res. 5, 438-446 (2019). 
24. Hurley, E. et al. Comparison of the salivary and dentinal microbiome of children with severe-early childhood caries to the salivary microbiome of caries-free children. BMC Oral Health https://doi.org/10.1186/s12903-018-0693-1 (2019).

25. Ortiz, S. et al. Sex-specific differences in the salivary microbiome of caries-active children. J. Oral Microbiol. https://doi. org/10.1080/20002297.2019.1653124 (2019).

26. Minty, M. et al. Gender-associated differences in oral microbiota and salivary biochemical parameters in response to feeding. J. Physiol Biochem. https://doi.org/10.1007/s13105-020-00757-x (2020).

27. Chong, J., Liu, P., Zhou, G. \& Xia, J. Using MicrobiomeAnalyst for comprehensive statistical, functional, and meta-analysis of microbiome data. Nat. Protoc. 15, 799-821 (2020).

28. Foster, Z. S., Sharpton, T. J. \& Grünwald, N. J. Metacoder: an R package for visualization and manipulation of community taxonomic diversity data. PLoS Comput. Biol. 13(2), e1005404 (2017).

29. Chen, C. et al. Oral microbiota of periodontal health and disease and their changes after nonsurgical periodontal therapy. ISME J. 12, 1210-1224 (2018).

30. Shi, W., Tian, J., Xu, H., Zhou, Q. \& Qin, M. Distinctions and associations between the microbiota of saliva and supragingival plaque of permanent and deciduous teeth. PLoS ONE 13, e0200337 (2018).

31. Ghani, N. A., Norizan, S. N. M., Chan, X. Y., Yin, W. F. \& Chan, K. G. Labrenzia sp. BM1: a quorum quenching bacterium that degrades N-acyl homoserine lactones via lactonase activity. Sensors 14, 11760-11769 (2014).

32. Jiang, S., Gao, X., Jin, L. \& Lo, E. C. M. Salivary microbiome diversity in caries-free and caries-affected children. Int. J. Mol. Sci. https://doi.org/10.3390/ijms17121978 (2016).

33. Demirjian, A. \& Levesque, G. Y. Sexual differences in dental development and prediction of emergence. J. Dent Res. 59, 1110-1122 (1980).

34. Cheng, Y. H., Liao, Y., Chen, D. Y., Wang, Y. \& Wu, Y. Prevalence of dental caries and its association with body mass index among school-age children in Shenzhen, China. BMC Oral Health 19, 270. https://doi.org/10.1186/s12903-019-0950-y (2019).

35. Ferraro, M. \& Vieira, A. R. Explaining gender differences in caries: a multifactorial approach to a multifactorial disease. Int. J. Dent. https://doi.org/10.1155/2010/649643 (2010).

36. Jagathrakshakan, S. N., Sethumadhava, R. J., Mehta, D. T. \& Ramanathan, A. 16S rRNA gene-based metagenomic analysis identifies a novel bacterial co-prevalence pattern in dental caries. Eur. J. Dent. 9, 127-132 (2015).

37. Xiao, C., Ran, S., Huang, Z. \& Liang, J. Bacterial diversity and community structure of supragingival plaques in adults with dental health or caries revealed by $16 \mathrm{~S}$ pyrosequencing. Front. Microbiol. https://doi.org/10.3389/fmicb.2016.01145 (2016).

38. Belstrøm, D. et al. Salivary microbiota in individuals with different levels of caries experience. J. Oral Microbiol. https://doi. org/10.1080/20002297.2016.1270614 (2017).

39. Eberspächer, J., Lingens, F. The genus Phenylobacterium. The prokaryotes. New York. https://doi.org/10.1007/0-387-30745-1_13 (2006).

40. Götz, F., Bannerman, T. \& Schleifer, K. H. The genera staphylococcus and macrococcus. The prokaryotes (Springer, New York, 2006). https://doi.org/10.1007/0-387-30744-3_1.

41. Liu, S., Wang, Y., Zhao, L., Sun, X. \& Feng, Q. Microbiome succession with increasing age in three oral sites. Aging 12, 7874 (2020).

42. Moynihan, P. Sugars and dental caries: Evidence for setting a recommended threshold for intake. Adv. Nutr. 7, 149-156 (2016).

43. Kuusela, S., Honkala, E., Kannas, L., Tynjala, J. \& Wold, B. Oral hygiene habits of 11-year-old schoolchildren in 22 European countries and Canada in 1993/1994. J. Dent Res. 76, 1602-1609 (1997).

44. Percival, R. S., Challacombe, S. J. \& Marsh, P. D. Flow rates of resting whole and stimulated parotid saliva in relation to age and gender. J. Dent. Res. 73, 1416-1420 (1994).

45. Lukacs, J. R. Sex differences in dental caries experience: clinical evidence, complex etiology. Clin. Oral Invest. 15, 649-656 (2011).

46. Lintula, T. et al. Self-reported oral health and associated factors in the North Finland 1966 birth cohort at the age of 31. BMC Oral Health https://doi.org/10.1186/1472-6831-14-155 (2014).

47. Mattila, A. et al. Self-reported oral health and use of dental services among asylum seekers and immigrants in Finland-a pilot study. Eur. J. Public Health. 26, 1006-1010 (2016).

48. Wise, G. E. \& King, G. J. Mechanisms of tooth eruption and orthodontic tooth movement. J. Dent Res. 87, 414-434 (2008).

49. Widström, E. \& Järvinen, S. Caries prevalence and use of dental services in Finnish children and adolescents in 2009. Oral. Health Dent. Manag. 10, 185-192 (2011).

50. Larmas, M. Has dental caries prevalence some connection with caries index values in adults?. Caries Res. 44, $81-84$ (2010).

51. Bernabe, E., Suominen-Taipale, A. L., Vehkalahti, M., Nordblad, A. \& Sheiham, A. The T-Health index: a composite indicator of dental health. Eur. J. Oral Sci. 117, 385-389 (2009).

52. Hajishengallis, G., Darveau, R. P. \& Curtis, M. A. The keystone-pathogen hypothesis. Nat. Rev. Microbiol. 10, 717-725 (2012).

53. Figueiredo, R. A. D. O. et al. Cohort profile: the Finnish health in teens (Fin-HIT) study: a population-based study. Int. J. Epidemiol. 48, 22-24H (2019).

54. Raju, S. C. et al. Antimicrobial drug use in the first decade of life influences saliva microbiota diversity and composition. Microbiome 8, 121. https://doi.org/10.1186/s40168-020-00893-y (2020).

55. Klein, H., Palmer, C. E. \& Knutson, J. W. Studies on dental caries: I. Dental status and dental needs of elementary school children. Public Heal Rep. 53, 751-765 (1938).

56. Ainamo, J. et al. Development of the World Health Organization (WHO) community periodontal index of treatment needs (CPITN). Int. Dent J. 32, 281-291 (1982).

57. Viljakainen, J. et al. Meal regularity plays a role in shaping the saliva microbiota. Front. Microbiol. 11, 757. https://doi.org/10.3389/ fmicb.2020.00757 (2020)

58. Eshriqui, I. et al. Breastfeeding may have a long-term effect on oral microbiota: results from the Fin-HIT cohort. Int. Breastfeed. J. 15, 42. https://doi.org/10.1186/s13006-020-00285-w (2020).

59. Raju, S. C. et al. Reproducibility and repeatability of six high-throughput $16 \mathrm{~S}$ rDNA sequencing protocols for microbiota profiling. J. Microbiol. Methods 147, 76-86 (2018).

60. Klindworth, A. et al. Evaluation of general $16 \mathrm{~S}$ ribosomal RNA gene PCR primers for classical and next-generation sequencingbased diversity studies. Nucleic Acids Res. https://doi.org/10.1093/nar/gks808 (2013).

61. Schloss, P. D. et al. Introducing mothur: Open-source, platform-independent, community-supported software for describing and comparing microbial communities. Appl. Environ. Microbiol. 75, 7537-7541 (2009).

62. Meyer, M. \& Kircher, M. Illumina sequencing library preparation for highly multiplexed target capture and sequencing. Cold Spring Harb. Protoc. https://doi.org/10.1101/pdb.prot5448 (2010).

63. Edgar, R. C., Haas, B. J., Clemente, J. C., Quince, C. \& Knight, R. UCHIME improves sensitivity and speed of chimera detection. Bioinformatics 27, 2194-2200 (2011).

64. Quast, C. et al. The SILVA ribosomal RNA gene database project: improved data processing and web-based tools. Nucleic Acids Res. 41, 590-596 (2012).

65. Dixon, P. VEGAN, a package of R functions for community ecology. J. Veg. Sci. 14, 927-930 (2003). 


\title{
Acknowledgements
}

We acknowledge all the children and parents who participated in the study and all fieldworkers for data collection and technical assistance.

\section{Author contributions}

Conceptualization: M.M., J.F., H.V.; Data curation: M.M., S.R., C.S., H.V.; Formal analysis: M.M., S.L., S.R., H.V.; Funding acquisition: M.M., C.S., H.V.; Investigation: M.M., S.L., S.R., H.V.; Methodology: M.M., J.F., S.L., E.E., H.V.; Project administration: C.S., E.E.; Supervision: H.V.; Visualization: M.M., S.L., H.V.; Writing—original draft: M.M., H.V.;Writing—review \& editing: M.M., S.L., J.F., C.S., E.E., S.R., H.V.

\section{Funding}

This project received financial support from the Academy of Finland (Grant No. 332459), the Swedish Cultural Foundation in Finland, the Päivikki and Sakari Sohlberg Foundation, the Minerva Foundation / Selma and Maja-Lisa Selander Fund, and the Folkhälsan Research Foundation. The funders had no role in study design, data collection and analysis, preparation of the manuscript or decision to publish.

\section{Competing interests}

The authors declare no competing interests.

\section{Additional information}

Supplementary Information The online version contains supplementary material available at https://doi. org/10.1038/s41598-021-83846-1.

Correspondence and requests for materials should be addressed to H.V.

Reprints and permissions information is available at www.nature.com/reprints.

Publisher's note Springer Nature remains neutral with regard to jurisdictional claims in published maps and institutional affiliations.

\begin{abstract}
Open Access This article is licensed under a Creative Commons Attribution 4.0 International License, which permits use, sharing, adaptation, distribution and reproduction in any medium or format, as long as you give appropriate credit to the original author(s) and the source, provide a link to the Creative Commons licence, and indicate if changes were made. The images or other third party material in this article are included in the article's Creative Commons licence, unless indicated otherwise in a credit line to the material. If material is not included in the article's Creative Commons licence and your intended use is not permitted by statutory regulation or exceeds the permitted use, you will need to obtain permission directly from the copyright holder. To view a copy of this licence, visit http://creativecommons.org/licenses/by/4.0/.
\end{abstract}

(C) The Author(s) 2021 